\title{
Differential expression and functions of Ehm2 transcript variants in lung adenocarcinoma
}

\author{
SHENGLAN LI ${ }^{1,2}$, JIAN MA $^{1,2}$, YANG SI $^{1,2}$, SHAN CHENG $^{1,2}$, MU HU $^{3}$, \\ XIUYI ZHI ${ }^{3}$, BAOLAN LI ${ }^{4}$, HEFEN YU ${ }^{1,2}$ and WEN G. JIANG ${ }^{1,2,5}$ \\ ${ }^{1}$ Department of Biochemistry and Molecular Biology, School of Basic Medical Sciences, Capital Medical University; \\ ${ }^{2}$ Beijing Key Laboratory of Cancer Invasion and Metastasis Research, Capital Medical University, Beijing 100069; \\ ${ }^{3}$ Department of Thoracic Surgery, Beijing Xuanwu Hospital, Capital Medical University, Beijing 100053; \\ ${ }^{4}$ Beijing Thoracic Tumor Research Institute, Beijing Chest Hospital, Capital Medical University, Beijing 101149, P.R. China; \\ ${ }^{5}$ Cardiff China Medical Research Collaborative, Cardiff University School of Medicine, Cardiff CF14 4XN, UK
}

Received July 9, 2018; Accepted January 4, 2019

DOI: $10.3892 /$ ijo.2019.4732

\begin{abstract}
Ehm2 [also known as erythrocyte membrane protein band 4.1-like protein 4B (EPB41L4B)] is a member of the NF2/ERM/4.1 superfamily. The overexpression of Ehm 2 has been observed in metastatic cancer cells. Through alternative splicing, the Ehm 2 gene produces two transcript variants that encode the two different isoforms, Ehm2/1 and Ehm2/2. The biological functions of these different Ehm 2 transcript variants remain unclear. The present study aimed to determine the expression of the Ehm 2 variants in lung adenocarcinoma and their involvement in the disease progression of the patients. The expression of Ehm 2 transcript variants in human lung adenocarcinoma tissues was analyzed using immunohistochemistry and western blot analysis. Ehm2 variants were overexpressed or knocked down in A549 human lung adenocarcinoma cells. The consequent effects of the genetic modifications on the cellular functions of lung cancer cells were then examined using in vitro cell viability, invasion and migration assays. The expression of epithelial-mesenchymal transition (EMT)-related markers was evaluated by western blot analysis in the cell models. The association of Ehm2 variant expression with patient survival was analyzed using Kaplan-Meier survival analysis. The expression of Ehm2/1 was significantly decreased in lung cancers compared with the paired normal lung tissues $(\mathrm{P}<0.05)$, while the Ehm $2 / 2$ protein
\end{abstract}

Correspondence to: Dr Hefen Yu, Department of Biochemistry and Molecular Biology, School of Basic Medical Sciences, Capital Medical University, 10 Youanmenwai, Xitoutiao, Fengtai, Beijing 100069, P.R. China

E-mail:yhf23@ccmu.edu.cn

Professor Wen G. Jiang, Cardiff China Medical Research Collaborative, Cardiff University School of Medicine, Heath Park, Cardiff CF14 4XN, UK

E-mail: jiangw@cf.ac.uk

Key words: Ehm2, lung adenocarcinoma, transcript variant, cell function, epithelial-mesenchymal transition levels were higher in the tumors than in the paired normal lung tissues, although this was not statistically significant. The overexpression of Ehm2/1 exerted inhibitory effects, while the knockdown of Ehm2/1 promoted the growth, invasion and migration of A549 cells in vitro. Ehm2/2 was expressed at low levels in the A549 cells and the enforced expression of Ehm 2/2 significantly increased the invasiveness and migration of the A549 cells. Immunofluorescence staining revealed that Ehm2/1 was confined to the plasma membrane, while Ehm2/2 was observed at both the plasma membrane and cytoplasm. The overexpression of Ehm2/1 resulted in the upregulation of the epithelial marker, E-cadherin, and in the decreased expression of the mesenchymal markers, N-cadherin and Snail1, while the knockdown of Ehm2/1 and the enforced expression of Ehm2/2 had the opposite effects on the protein levels of EMT-related markers. Kaplan-Meier survival analysis revealed that higher Ehm2/1 transcript levels were associated with the longer survival of patients with lung adenocarcinoma, while the lower expression of Ehm2/2 exhibited a similar association with patient survival. Taken together, the two Ehm 2 variants appear to be differentially expressed in lung adenocarcinoma. Ehm2/1 may function as a putative tumor suppressor in the disease progression of lung adenocarcinoma, while Ehm 2/2 may have an opposite function.

\section{Introduction}

To date, lung cancer remains as the leading type of cancer according to its incidence and mortality rate worldwide (1). It comprises two main histological subtypes, non-small cell lung cancer (NSCLC) and small-cell lung cancer (SCLC). Approximately $85 \%$ of lung cancer cases are NSCLC, which includes squamous cell carcinomas, adenocarcinomas and large cell carcinomas (2). The incidence of lung adenocarcinoma continues to increase in developed countries. It has become the most common subtype of lung cancer for both smokers and lifelong non-smokers. However, relapse is commonly observed in 50 to $70 \%$ of patients within 1 year following surgical therapy, which is closely associated with the nature and quality of the surgery (3). Patients with NSCLC carrying 
mutated epidermal growth factor receptor (EGFR) exhibit a good response to tyrosine kinase inhibitor (TKI) (4). However, resistance to TKIs acquired by cancer cells often limits the effects and corresponding benefits (5). Therefore, intensive research is required to shed light on the molecular and cellular machinery which are 'hijacked' or acquired by the lung cancer cells during tumor development and disease progression.

Ehm2, also known as erythrocyte membrane protein band 4.1-like protein 4B (EPB41L4B), belongs to the Four.1 protein, ezrin, radixin, moesin (FERM) superfamily. The overexpression of Ehm 2 has been observed in metastatic cancer cells (6). FERM proteins contain a highly conserved FERM domain which mediates protein-protein interactions through an interaction with the cytoplasmic tails of transmembrane proteins $(7,8)$. FERM proteins are cytoskeletal-associated proteins mediating interactions between transmembrane proteins and the cytoskeletal proteins. Upon an interaction with Crb3 through its FERM-binding motif, Ehm2 can be recruited to the plasma membrane, leading to an activation of p114RhoGEF and the consequential control of the morphology and cohesion of cancer cells (9). Lulu2, a murine Ehm 2 protein, has been shown to activate cortical myosin II to alter contractile force in epithelial cells (10). In human fibrosarcoma cells, Ehm2 has been reported to be involved in steroid-regulated cytoskeletal reorganization (11).

As a metastasis-associated protein, Ehm2 is frequently dysregulated in human solid cancers $(6,12)$. The upregulation of Ehm 2 has been observed in both tumor tissues and cell lines of prostate cancer (13). The overexpression of Ehm 2 can promote prostate cancer progression and metastasis (13). The expression of Ehm 2 is also elevated in breast cancers, which is associated with metastasis and a poor prognosis of patients with the disease (14). The knockdown of Ehm2 in MCF-7 cells has been shown to not only increase apoptosis, but also to reduce invasiveness through a regulation of matrix metalloproteinase (MMP)9 (14).

Alternative splicing is a regulated process in gene expression, which makes a single gene to encode different protein products. It naturally occurs in approximately $95 \%$ of multi-exonic genes in eukaryotes $(15,16)$, and is also involved in various disorders including cancers (17-21). Through alternative splicing, the Ehm2 gene produces two transcript variants, encoding two different isoforms, Ehm $2 / 1$ and Ehm2/2. The isoform 1 has 382 amino acids missing in its C-terminal region in comparison with Ehm2/2 (11). Although the involvement of Ehm2 in disease progression is evident in both prostate and breast cancers, as discussed above, the exact role and function played by the two splice variants have yet to be elucidated.

In the present study, we examined the expression of the two Ehm 2 transcript variants in lung adenocarcinoma tissues. The two variants exhibited distinct patterns of expression in lung cancers. In vitro cell function assays revealed that Ehm $2 / 1$ exerted inhibitory effects, and that Ehm $2 / 2$ promoted both the migration and invasion of A549 cells. We also examined the association of the Ehm2 variants with epithelial-mesenchymal transition (EMT) in lung cancer cells.

\section{Materials and methods}

Cells and cell culture. The A549 cell line was purchased from the American Type Culture Collection (ATCC, Manassas,
VA, USA). The cells were cultured using Dulbecco's modified Eagle's medium (DMEM)-F12 (Thermo Fisher Scientific, Waltham, MA, USA) which was routinely supplemented with $10 \%$ fetal bovine serum (Thermo Fisher Scientific) and penicillin/streptomycin (KGY0023; KeyGEN BioTECH, Nanjing, China) in an incubator at $37^{\circ} \mathrm{C}$ with $5 \% \mathrm{CO}_{2}$ and $95 \%$ humidity.

Human lung cancer specimens. Fresh-frozen NSCLC tissues $(\mathrm{n}=15)$ and matched adjacent normal lung tissues from the same patients were collected immediately following surgical resection at Xuanwu Hospital of Capital Medical University (Beijing, China) after obtaining written informed consent. These tissues were stored at $-80^{\circ} \mathrm{C}$ until use. Clinicopathological information was collected and recorded in a database which includes sex, age, TNM stage, histological tumor type and lymph node metastasis. The clinicopathological characteristics of these tumor cases are presented in Table SI. All protocols were reviewed and approved by the Ethics Committee of Xuanwu Hospital.

Immunohistochemistry using tissue microarrays. Tumor tissue microarrays (TMA: HLug-Ade060PG-01) were purchased from Shanghai Outdo Biotechnology Co., Ltd. Each tissue microarray contained paraffin-embedded sections from 30 lung adenocarcinoma tissues, as detailed in Table I, together with the paired normal tissues. Pathological diagnosis for these lung adenocarcinomas was available in the manufacturer's instructions. Immunohistochemical staining of tissue microarrays was performed as follows: After being dewaxed for $2 \mathrm{~h}$ at $60^{\circ} \mathrm{C}$ and rehydrated by a gradient method, antigen retrieval was performed using a citrate buffer (10 mM citric acid, $\mathrm{pH}$ 6.0) followed by a blocking in a blocking buffer containing 5\% bovine serum albumin. Primary antibodies against human Ehm2/1 (1:200; AP338037; OriGene Technologies, Inc., Rockville, MD, USA) and human Ehm2/2 (1:50; ab135616; Abcam, Cambridge, UK) were used for probing (incubation for $20 \mathrm{~min}$ at room temperature). A negative control was included without the addition of any primary antibodies. The sections were subsequently incubated with the relevant secondary antibody (ZSGB Biotechnology, Beijing, China) for $20 \mathrm{~min}$ at room temperature. Microarrays were visualized using diaminobenzidine (Cell Signal Technology, Inc., Danvers, MA, USA) with a counterstaining of nuclei using hematoxylin. All these processes were automatically carried out in a fully automated immunohistochemistry and in situ hybridization system (Leica BOND-MAX; Leica Biosystems, Richmond, IL, USA).

Semi-quantitative analysis of the intensity of Ehm2/1 and Ehm2/2 immunochemical staining in tissue microarrays. All immunochemical staining images in the tissue microarrays were acquired using a microscope which equipped with a digital camera (BX43; Olympus, Tokyo, Japan). The staining was assessed by two independent investigators who were blinded to the clinical information. The integrated optical density (IOD) of Ehm2/1 and Ehm2/2 staining in the tissue microarrays was determined using ImagePro ${ }^{\circledR}$ Plus software version 5.1 (Media Cybernetics, Inc., Rockville, MD, USA). 
Table I. Clinicopathological characteristics of the 30 tumor cases.

\begin{tabular}{|c|c|c|c|}
\hline Parameters & Category & Cases, $n=30$ & Percentage $(\%)$ \\
\hline \multirow[t]{2}{*}{ Tissue paired samples } & Tumor & 30 & \\
\hline & Normal & 30 & \\
\hline \multirow[t]{2}{*}{ Lymph node status } & No & 4 & 13 \\
\hline & $\mathrm{N}>1$ & 26 & 86 \\
\hline \multirow[t]{3}{*}{ Pathological grades } & $\mathrm{I}$ & 5 & 17 \\
\hline & II & 19 & 63 \\
\hline & III & 6 & 20 \\
\hline \multirow[t]{4}{*}{ TNM staging } & TNM I & 3 & 10 \\
\hline & TNM II & 14 & 47 \\
\hline & TNM III & 13 & 43 \\
\hline & TNM IV & 0 & 0 \\
\hline \multirow[t]{3}{*}{ Age, years } & $\leq 40$ & 3 & 10 \\
\hline & $41-56$ & 6 & 20 \\
\hline & $\geq 56$ & 21 & 70 \\
\hline Mean \pm SD & $57.11 \pm 10.09$ & & \\
\hline \multirow[t]{2}{*}{ Sex } & Male & 11 & 37 \\
\hline & Female & 19 & 63 \\
\hline \multirow[t]{2}{*}{ Pathological types } & Adenocarcinoma & 30 & 100 \\
\hline & Squamous cell carcinoma & 0 & 0 \\
\hline
\end{tabular}

$\mathrm{SD}$, standard deviation.

The average IOD/Ehm2 positively stained area $\left(\mu \mathrm{m}^{2}\right)$ was calculated for the positively stained tissues.

Cell transfection. Expression plasmids of Ehm2/1 (RC223085) and Ehm2/2 (RC212424) tagged with FLAG were purchased from OriGene Technologies, Inc.. The shRNA targeting Ehm2/1 was purchased from Genechem Co. (Shanghai, China). Plasmid transfections were performed using jetPRIME ${ }^{\circledR}$ transfection reagent (Polyplus Transfection, Illkirch, France) according to the manufacturer's instructions. The cells were harvested for an analysis of gene expression approximately $48 \mathrm{~h}$ following transfection. A549 wild-type (A549 cells not transfected with any plasmid) and empty vector transfected (pCMV-Entry) cells were used as controls.

Western blot analysis. The cells were lysed with a RIPA lysis buffer. Protein extracts were prepared from frozen tissues as follows. Tissues were homogenized using a protein

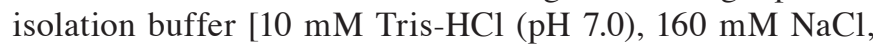
$1 \%$ Triton X-100, 1 mM EGTA, 1 mM EDTA, and Roche complete protease inhibitors]. The samples were incubated for $15 \mathrm{~min}$ on ice followed by a centrifugation at $16,000 \mathrm{x} \mathrm{g}$ for $30 \mathrm{~min}$. The supernatants were stored at $-80^{\circ} \mathrm{C}$ until use. A BCA protein assay kit (Thermo Fisher Scientific) was used to determine the protein concentration. A total of $25 \mu \mathrm{g}$ of protein samples were separated by sodiumdodecyl sulfate-polyacrylamide gel electrophoresis followed by an electric blotting onto polyvinylidenedifluoride membranes (Merck Millipore, Billerica, MA, USA). Protein was probed with primary antibodies against Ehm2/1 (1:1,000; AP33087; OriGene Technologies, Inc.), Ehm2/2 (1:500; ab135616; Abcam), FLAG (1:1,000; F3165; Sigma, St. Louis, MO, USA), E-cadherin (1:1,000; ab40772; Abcam), N-cadherin (1:1,000; ab19348; Abcam), Snail1 (1:2,000; ab53519; Abcam), GAPDH (1:3,000; sc-32233; Santa Cruz Biotechnology, Inc., Santa Cruz, CA, USA), at $4^{\circ} \mathrm{C}$ overnight. The horseradish peroxidase-conjugated goat anti-mouse antibody (1:5,000; 115-035-003; Jackson ImmunoResearch Laboratories, Inc., West Grove, PA, USA) and goat anti-rabbit antibody (1:5,000; 111-035-03; Jackson ImmunoResearch Laboratories, Inc.) were incubated with the membranes for $1 \mathrm{~h}$ at room temperature. Protein bands were documented using a chemiluminescence (ECL) detection system (FusionFx; VilberLourmat, Collégien, France). Protein expression was determined by densitometric analysis using Image $\mathrm{J}$ software (version 1.62; National Institutes of Health, Bethesda, MD, USA). Quantitative densitometric values of all proteins were normalized to GAPDH.

In vitro cell viability assay. The cells were seeded into 96-well plates at a density of $3 \times 10^{3}$ cells per well. Cell viability was then evaluated using a Cell Counting Kit-8 (CCK8; Dojindo, Kumamoto, Japan). Absorbance was determined at a wavelength of $450 \mathrm{~nm}$ using a spectrophotometer (BioTek, Winooski, VT, USA).

Wound healing assay. The cells were plated into 12-well plates at a density of $6 \times 10^{5}$ cells per well and allowed to 
form a monolayer. The cells were wounded with a fine gauge needle to create a wound. The migration of the cells was recorded using an inverted microscope (CKX41; Olympus Corp., Tokyo, Japan). Images were captured using a camera (EOS600D; Canon, Tokyo, Japan) at the indicated time point. The migration distances were determined and analyzed using Image J software (version 1.62).

In vitro invasion assay. Matrigel (BD Biosciences, San Jose, CA, USA) was used to pre-coat Transwell cell culture inserts ( $8 \mu \mathrm{m}$ pore size, 28617043; Costar, New York, NY, USA) and air dried. Following rehydration, 20,000 cells were seeded into each insert. Following 2 days of incubation, the invaded cells were fixed in $4 \%$ formalin, followed by staining with $0.5 \%$ crystal violet (0528; Amresco Inc., Solon, OH, USA) for $15 \mathrm{~min}$ at room temperature. The absorbance was measured at $490 \mathrm{~nm}$ using a spectrophotometer (BioTek).

Gelatin zymography assay. The activities of MMPs were assayed as previously described (14). The cells were seeded into tissue culture flasks at a density of $1 \times 10^{6}$ cells. Once the cells reached $80 \%$ confluency, they were washed with phosphate-buffered saline (PBS) and further cultured in serum-free medium. After $6 \mathrm{~h}$, conditioned medium was collected and concentrated through an Amicon Ultra-0.5 $\mathrm{ml}$ centrifugal filter (Merck Millipore, East Midlands, UK). Protein samples were prepared using a non-reducing sample buffer. Protein samples were separated via 10\% SDS-PAGE containing $0.1 \%$ gelatin. Following electrophoresis, the gels were washed and incubated overnight at $37^{\circ} \mathrm{C}$. Following incubation, the gels were stained with Coomassie blue and subsequently scanned on digital scanner images (HP Scanjet G3110; HP Development Co., Beijing, China).

Immunofluorescence staining of cells. The cells were seeded on coverslips. Following fixation with $4 \%$ paraformaldehyde, the cells were washed 3 times with ice-cold phosphate-buffered saline (PBS), and then permeabilized for $30 \mathrm{~min}$. The cells were then incubated with anti-FLAG (1:100; F3165; Sigma) antibody at room temperature for $1 \mathrm{~h}$ followed by further $1 \mathrm{~h}$ of incubation at room temperature with Alexa Fluor ${ }^{\circledR}$ 488-conjugated goat anti-mouse secondary antibody (1:200; A11029; Thermo Fisher Scientific). The slides were mounted using ProLong ${ }^{\circledR}$ Gold Antifade Mountant with DAPI (P36931; Thermo Fisher Scientific). A Leica SP8 confocal microscope (Leica Microsystems, Wetzlar, Germany) was used to photograph the slides.

$R N A$ preparation and reverse transcription-polymerase chain reaction $(R T-P C R)$. RNA extraction and RT-PCR were performed as previously described (22). Total RNA was isolated from frozen lung cancer tissues and lung adenocarcinoma A549 cells using TRIzol ${ }^{\circledR}$ reagent (15596018; Thermo Fisher Scientific). cDNA was generated from $1 \mu \mathrm{g}$ RNA sample using an iScript ${ }^{\mathrm{TM}}$ cDNA Synthesis kit (1708891; Bio-Rad Laboratories, Inc., Hercules, CA, USA). The reaction conditions for reverse transcription were as follows: $25^{\circ} \mathrm{C}$ for $5 \mathrm{~min}, 42^{\circ} \mathrm{C}$ for $30 \mathrm{~min}$, and $85^{\circ} \mathrm{C}$ for $5 \mathrm{~min}$. PCR was carried out using a REDTaq $^{\text {TM }}$ ReadyMix PCR reaction mix (R2523; Sigma) according to the manufacturer's instructions. The reaction conditions for PCR were as follows: $94^{\circ} \mathrm{C}$ for $5 \mathrm{~min}$ for initial denaturation, followed by 30 cycles of $94^{\circ} \mathrm{C}$ for $10 \mathrm{sec}, 56^{\circ} \mathrm{C}$ for $20 \mathrm{sec}$ and $72^{\circ} \mathrm{C}$ for $30 \mathrm{sec}$. The PCR primers were as follows: Ehm 2/1 sense, 5'-CACTTTGAGAGACTGAAGCA-3' and antisense, 5'-CAACTTCTACGACAGGAATATATGC-3'; Ehm2/2 sense, 5'-CCTGTTGCGGATCATGTGAAGTG-3' and antisense, 5'-TATCAGGAAACGGGTTCATTGTATC-3'; and GAPDH sense, 5'-GGGAAGGTGAAGGTCGGAGT-3' and antisense, 5'-TTGAGGTCAATGAAGGGGT-3'. The products were visualized on a $1.2 \%$ agarose gel stained with GoldView (G8140; Solarbio, Beijing, China).

Quantitative polymerase chain reaction ( $q P C R$ ). qPCR was performed utilizing the ABI 7500 Fast Real-time PCR system (Applied Biosystems, Foster City, CA, USA) through the measurement of real-time SYBR-Green fluorescence (1708880; Bio-Rad Laboratories, Inc.), and the results were obtained by means of the comparative Cq method $\left(2^{-\Delta \Delta \mathrm{Cq}}\right)$ using GAPDH as an internal control (23). This experiment was performed in triplicate. The following condition was used in the reaction: $94^{\circ} \mathrm{C}$ for $5 \mathrm{~min}$ for initial denaturation, followed by 40 cycles of $94^{\circ} \mathrm{C}$ for $10 \mathrm{sec}, 56^{\circ} \mathrm{C}$ for $20 \mathrm{sec}$ and $72^{\circ} \mathrm{C}$ for $30 \mathrm{sec}$. The PCR primers were as follows: Ehm2/1 sense, 5'-TGAAGGTCGCATTGGAAAG-3' and antisense, 5'-CAT CATACTTTGCATCCCTCC-3'; Ehm2/2 sense, 5'-GTTGCG GATCATGTGAAGTG-3' and antisense, 5'-TGATTCCTC CTTTCCACCCT-3'; GAPDH sense, 5'-CTGAGTACGTCG GGAGTC-3' and antisense, 5'-GAGATGATGACCCTTTTG-3'.

Lung cancer survival analysis. The overall survival (OS), progression-free survival (PFS) and post-progression survival (PPS) of the patients with lung cancer was carried out using the web tool Kaplan-Meier Plotter (http://kmplot.com/analysis/). The Ehm2/1and Ehm2/2 probe sets were 220161_s_at and 233098_s_at, respectively.

Comparision of sequences of Ehm2/1 and Ehm2/2 in Ensemble and UCSC. Ensemble (http://asia.ensembl.org) is a genome browser for vertebrate genomes that supports research in comparative genomics, evolution, sequence variation, and so on. We queried Ehm2 (EPB41L4B) in human and found two transcripts for protein coding. UCSC Genome Browser (http://genome.ucsc.edu) can also provide the information of the Ehm2 transcript variants through the nearest mirror-genome-asia.ucsc.edu (http://genome-asia.ucsc.edu).

Statistical analysis. The SPSS software package version 19.0 (SPSS, Inc., Chicago, IL, USA) was used for the statistical analyses. Statistical analysis was performed using ANOVA and a Bonferroni post hoc test for multiple group comparisons. $\mathrm{P}<0.05$ was considered to indicate a statistically significant difference.

\section{Results}

Differential expression of Ehm2/1 andEhm2/2 in human lung adenocarcinoma specimens. To analyze the protein levels of Ehm 2 variants in lung cancer, the immunohistochemical staining of Ehm2/1 and Ehm2/2 was performed on tissue microarrays which comprised 30 human lung adenocarcinoma 
A
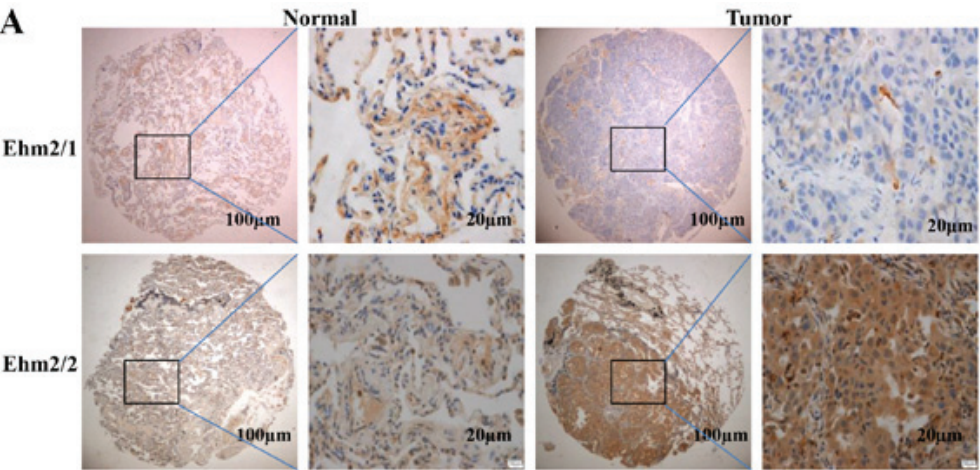

B
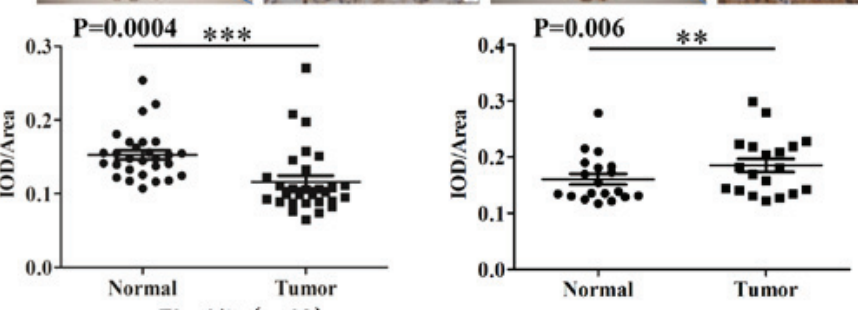

Ehm2/1 $\quad(n=28)$

Ehm2/2 (n=28)

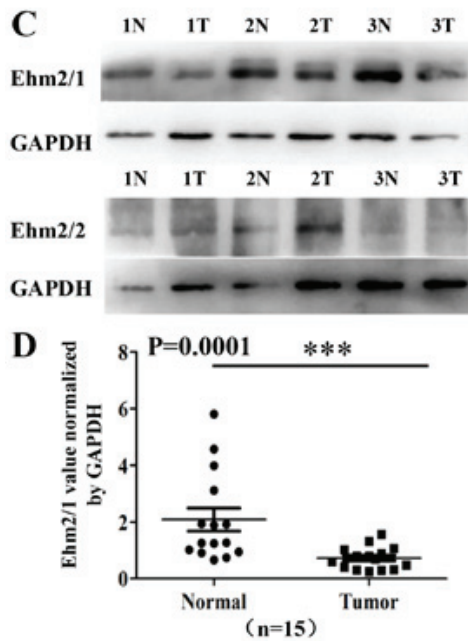

$\begin{array}{llllllllll}4 \mathrm{~N} & 4 \mathrm{~T} & 5 \mathrm{~N} & 5 \mathrm{~T} & 6 \mathrm{~N} & 6 \mathrm{~T} & 7 \mathrm{~N} & 7 \mathrm{~T}\end{array}$
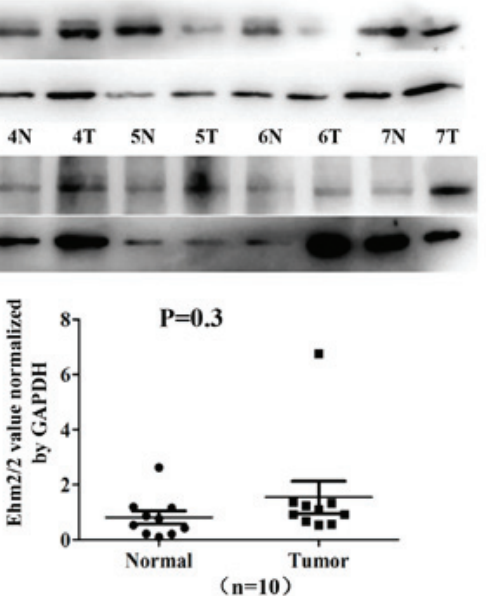

Figure 1. The two Ehm2 transcript variants were differentially expressed in human lung cancers. (A) Representative immunohistochemical staining of Ehm2/1 and Ehm2/2 in lung adenocarcinoma tissue microarrays. The square boxes in the images on the left panels showing the 'Normal' and 'Tumor' tissues are higher magnification images (cale bar, $100 \mu \mathrm{m}$ ) of the images on the right of these panels (scale bar, $20 \mu \mathrm{m}$ ). (B) Semi-quantitative integrated optical density of Ehm2/1-and Ehm2/2-positive staining was assayed in tissue microarrays. ${ }^{* * *} \mathrm{P}<0.01$ and ${ }^{* * * *} \mathrm{P}<0.001$. (C) Ehm2/1 and Ehm2/2 expression were determined in protein samples derived from human lung cancer tissues ( $T$ ) together with paired adjacent normal lung tissues (N). (D) Semi-quantitative analyses of Ehm2/1 and Ehm $2 / 2$ expression in (C) were performed using ImageJ software. The quantification of protein levels relative to GAPDH is shown. ${ }^{* * *} \mathrm{P}<0.001$.

tissues paired with non-cancerous tissues. Two tissue sections were invalid due to mounting issues and were excluded from the analyses. All information regarding the microarray tumor specimens is presented in Table I. The staining for Ehm2/1 was significantly decreased in the lung adenocarcinoma tissues compared with the paired normal tissues $(\mathrm{P}=0.0004, \mathrm{n}=28$; Fig. 1A and B), while the increased expression of Ehm2/2 was observed in comparison to the paired normal tissues $(\mathrm{P}=0.0060, \mathrm{n}=28$; Fig. $1 \mathrm{~A}$ and $\mathrm{B})$. We also determined the protein expression of these two Ehm 2 variants in lung cancer tissues by western blot analysis. The normal lung tissues predominantly expressed Ehm2/1 (Fig. 1C). Similarly, Ehm2/1 expression was markedly decreased in lung cancer tissues in comparison with the controls $(\mathrm{P}<0.01, \mathrm{n}=15$; Fig. 1D). Ehm $2 / 2$ was found to be upregulated in the lung adenocarcinoma tissues compared with the adjacent normal tissues, but this did not reach a level of significance ( $P=0.3, n=10$; Fig. 1D).
Our attempts to quantitatively analyze the mRNA expression levels of Ehm2/1 and Ehm $2 / 2$ in human samples using quantitative PCR were not successful owing largely to the fact that an insufficient quantity of mRNA isolated from these tissues was not adequate for the assay. Thus, it is clear from the above-mentioned immunohistochemistry results that the expression of Ehm $2 / 2$ was significantly increased in the lung adenocarcinomas $(\mathrm{P}=0.006)$. Taken together, from the results of immunohistochemistry and western blot analysis, it is clear that the expression of the two Ehm 2 transcript variants differs in human lung adenocarcinoma.

Ehm2/1 regulates the proliferation, migration and invasion of A549 cells in vitro. To examine the function of Ehm2/1 in lung adenocarcinoma, the human lung adenocarcinoma cell line, A549, was used to create an overexpression cell model. The endogenous expression of Ehm 2 in the A549 cells is 
$\mathbf{A}$
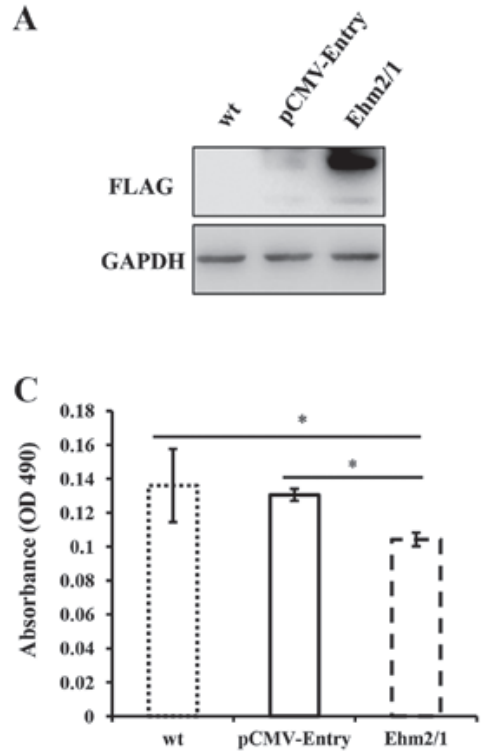

E

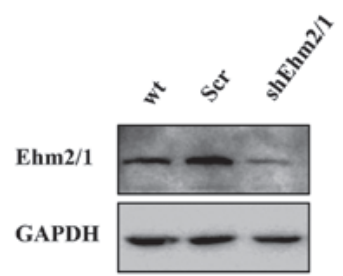

G

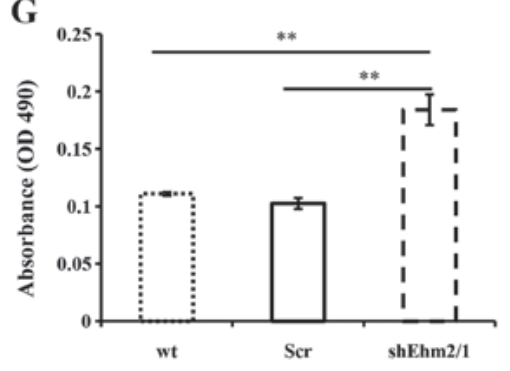

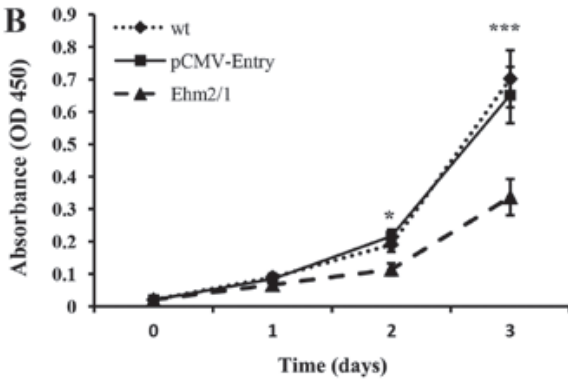
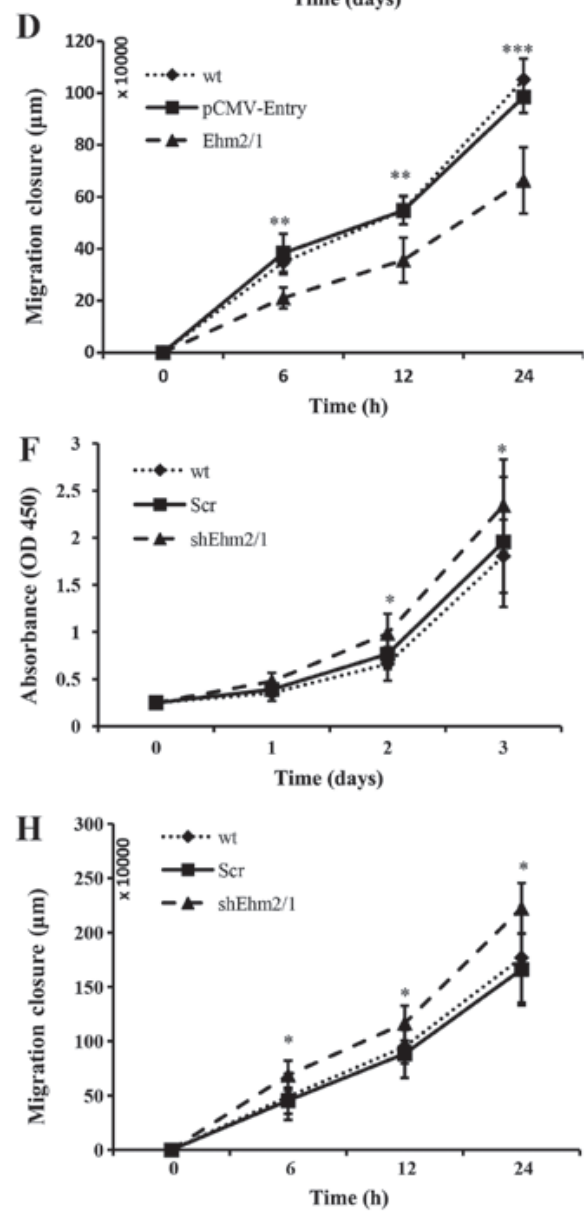

Figure 2. Ehm2/1 inhibits the biological functions of A549 human lung adenocarcinoma cells. (A) The overexpression of Ehm2/1 in A549 cells (Ehm2/1) was validated by western blot analysis. (B) A549 cells transfected with control vector and Ehm2/1 plasmid were subjected to cell viability assay. Shown are representative data from 3 independent experiments. Error bars represent standard deviations. ${ }^{* * *} \mathrm{P}<0.001$ when compared with pCMV-Entry control. (C) A549 cells transfected with control vector and Ehm $2 / 1$ plasmid were subjected to Transwell insert invasion assay. Invaded cells were stained and measured. Shown were representative data from 3 independent experiments. Error bars represent standard deviations. ${ }^{*} \mathrm{P}<0.05$. (D) A549 cells transfected with control vector and Ehm2/1 plasmid were wounded and the migration of cells was recorded. Shown are representative data from 3 independent experiments. Error bars represent standard deviations. ${ }^{* *} \mathrm{P}<0.01$ and ${ }^{* * *} \mathrm{P}<0.001$ when compared with pCMV-Entry. (E) Knockdown of Ehm2/1 in A549 cells (shEhm2/1) was confirmed by western blot analysis. Scr represents the empty vector control. (F-H) A549 cells transfected with control vector and Ehm2/1 shRNA were subjected to (F) cell viability, $(\mathrm{G})$ invasion and $(\mathrm{H})$ migration assays. Representative data are shown from 3 independent experiments. Error bars represent standard deviations. ${ }^{*} \mathrm{P}<0.05$ and ${ }^{* *} \mathrm{P}<0.01$ when compared with empty vector-transfected cells. pCMV-Entry represents the empty vector control, and wt represents A549 wild-type cells.

shown in Fig. S1. Western blot analysis was performed to verify the expression of Ehm2/1 in the transfected cells (Fig. 2A). Compared with the A549 wild-type (A549 cells not transfected with any plasmid) and empty vector-transfected (pCMV-Entry) control cells, the overexpression of Ehm2/1 exerted an inhibitory effect on cell proliferation in vitro $(\mathrm{P}<0.001$, when compared with pCMV-Entry control; Fig. 2B). Ehm2/1 overexpression markedly reduced the malignant phenotype of A549 cells, including invasiveness and migration $(\mathrm{P}<0.05$ and $\mathrm{P}<0.01$, respectively, when compared with pCMV-Entry; Fig. 2C and D). A knockdown model of Ehm2/1 in the A549 cells was also employed to verify the findings. A decreased expression of Ehm2/1 was observed in the cells in which Ehm2/1 was knocked down (shEhm2/1) compared with the A549 wild-type and empty vector-transfected cells (Scr) (Fig. 2E). The knockdown of Ehm2/1 expression resulted in a significant increase in cell growth in vitro $(\mathrm{P}<0.05$, when compared with empty vector-transfected cells; Fig. $2 \mathrm{~F})$, invasion $(\mathrm{P}<0.01$, when compared with empty vector-transfected cells; Fig. 2G) and 
A
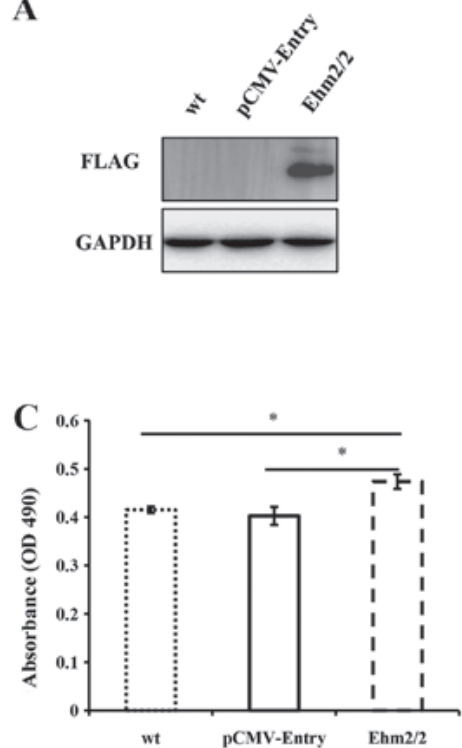
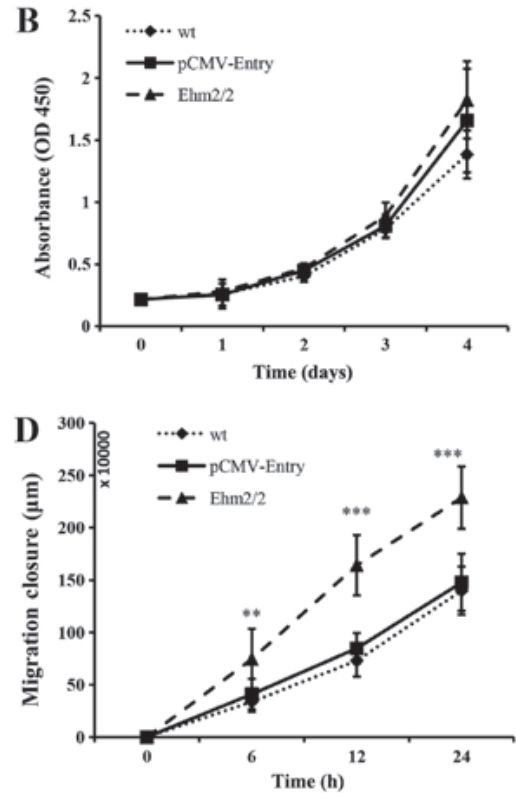

Figure 3. Overexpression of Ehm2/2 promotesthe biological functions of A549 human lung adenocarcinoma cells. (A) The expression of Ehm2/2 in A549 cells (Ehm2/2) was determined by western blot analysis. (B) A549 cells transfected with control vector and Ehm2/2 plasmid were subjected to cell viability assay. Shown are representative data from 3 independent experiments. Error bars represent standard deviations. (C) A549 cells transfected with control vector and Ehm2/2 plasmid were subjected to invasion assay. Invaded cells were stained and measured. Shown are representative data from 3 independent experiments. Error bars represent standard deviations. * $\mathrm{P}<0.05$. (D) A549 cells transfected with control vector and Ehm $2 / 2$ plasmid were wounded and the migration of cells was recorded. Three independent experiments were performed. Shown are representative data. Error bars represent standard deviations. ${ }^{* *} \mathrm{P}<0.01$ and ${ }^{* * *} \mathrm{P}<0.001$ when compared with pCMV-Entry control.

cell migration of the A549 cells $(\mathrm{P}<0.05$, when compared with empty vector-transfected cells; Fig. $2 \mathrm{H}$ ).

The invasive potential of the cancer cells is linked to their ability to degrade the extracellular matrix, which to a large degree is catalyzed by MMPs. Thus, in this study, we further determined the activities of MMPs from the cells with the differential expression of Ehm2 variants, using gelatin zymography assay. There was a marked increase in the activities of MMPs in the A549 cells in which Ehm2/1 was knocked down (Fig. S2). These findings indicate that Ehm2/1 is a negative regulator of A549 cell viability, invasion and migration.

Ehm2/2 expression promotes A549 cell invasion and migration. An Ehm2/2 overexpression cell line model was also generated to examine the regulatory role of this molecule in lung cancer cells. The expression level of Ehm2/2 was found to be considerably elevated in the Ehm2/2-overexpressing cells (Fig. 3A). The overexpression of Ehm $2 / 2$ was found to promote the proliferation of the A549 cells in comparison with the wild-type cells and empty vector-transfected control cells, although this difference was not statistically significant (Fig. 3B). In comparison to the control groups, the overexpression of Ehm 2/2 markedly increased the invasion of the A549 cells $(\mathrm{P}<0.05$, when compared with pCMV-Entry control; Fig. 3C). The ectopic expression of Ehm $2 / 2$ also markedly promoted cell migration $(\mathrm{P}<0.01$, when compared with pCMV-Entry control; Fig. 3D). In addition, gelatin zymography assay also revealed an increase in the activities of MMPs in the Ehm2/2-overexpressing cells (Fig. S2). Taken together, these data suggest that Ehm2/2 is a promoter of A549 cell invasion and migration.
Subcellular distribution of Ehm2/1 and Ehm2/2 differs in A549 cells. From the Ensemble and UCSC websites, we identified that Ehm2/1 variant lacks several exons and has a different 3'-terminal exon (data not shown). This results in Ehm2/2 with a long C-terminus and makes it longer than Ehm2/1. FERM proteins have been reported to have additional protein functions in the $\mathrm{C}$-terminal region, including an autoregulatory function that inhibits FERM domain activity $(8,24)$. Although thus far no functional domain has been found based on protein sequence alignment in the C-terminal region of Ehm2/2, at least to the best of our knowledge, we hypothesized that this C-terminal region may be important for its function and subcellular distribution through interacting with its FERM domain or with other proteins. Combined with differential expression patterns and differential effects on A549 cell function, we hypothesized that Ehm 2 variants have distinct subcellular distributions. To examine this possibility, we performed immunofluorescence staining of the A549 cells following the ectopic overexpression of Ehm2/1 and Ehm2/2. As shown in Fig. 4, Ehm2/1 was mainly found in the cell membrane (Fig. 4A), while Ehm2/2 was more widely distributed from the cell membrane, the cytoplasm and was even found in the nuclei (Fig. 4B). These results suggest that Ehm 2 variants have different distributions.

Ehm2/1 and Ehm2/2 influence EMT-marker expression in an opposing manner. It is known that the process of EMT enables cell migration and invasion. In consideration that Ehm2 variants regulate the invasion and migration of A549 cells, in this study, we detected the effects of Ehm2/1 and Ehm2/2 on EMT marker expression. The ectopic expression of Ehm2/1 resulted in the decreased expression of N-cadherin and Snaill, 
A

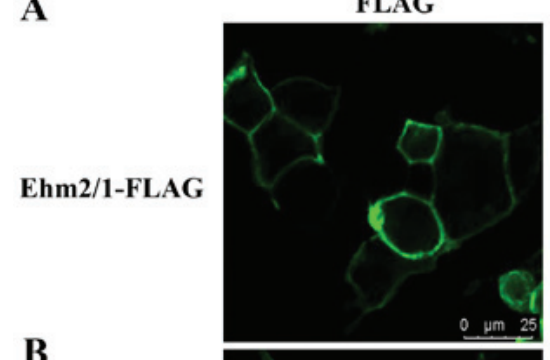

B

Ehm2/2-FLAG

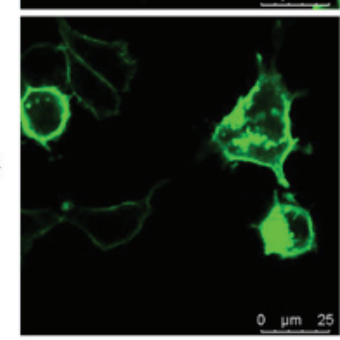

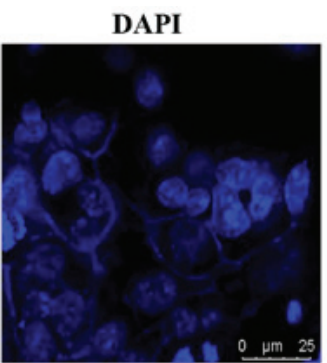
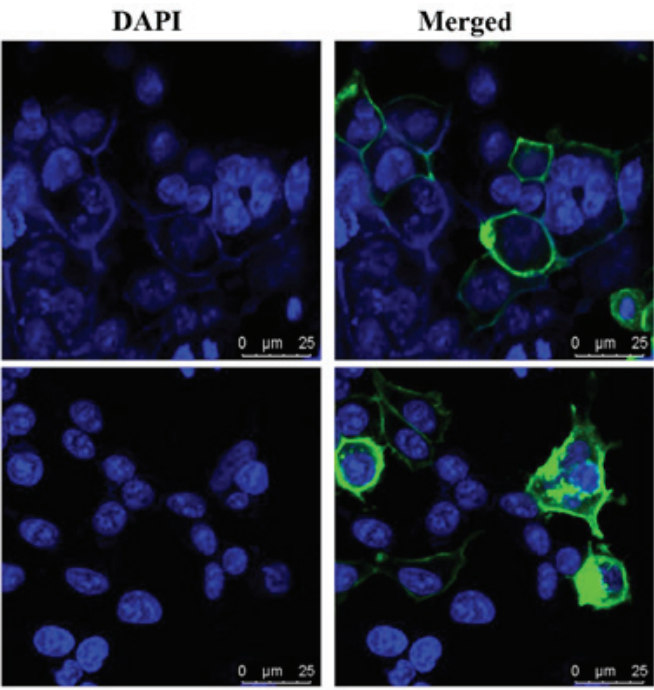

Figure 4. Subcellular distribution of Ehm2 variants in A549 cells. Immunofluorescence staining for Ehm2/1 and Ehm2/2 (green) and nuclei (DAPI; blue) were performed using A549 cells overexpressing (A) Ehm2/1 and (B) Ehm2/2.

$\mathbf{A}$

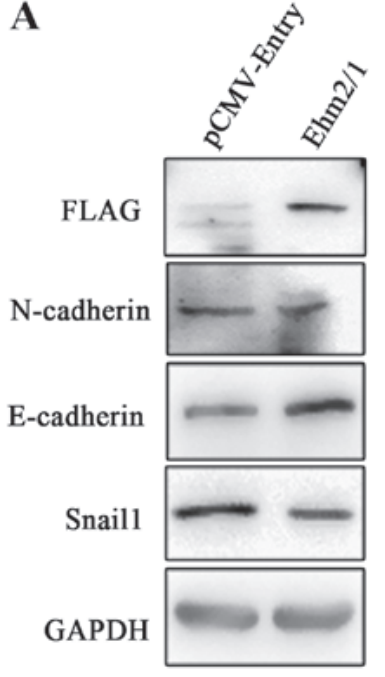

B

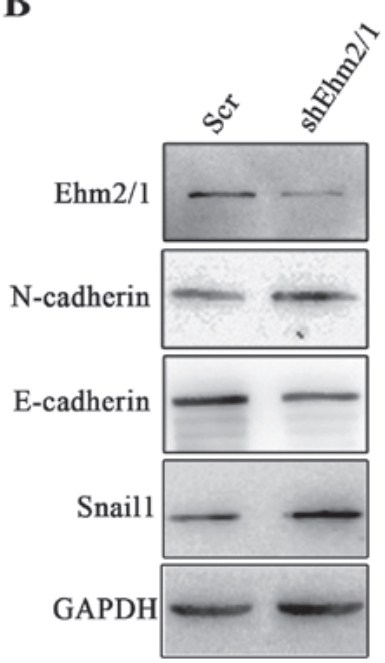

C

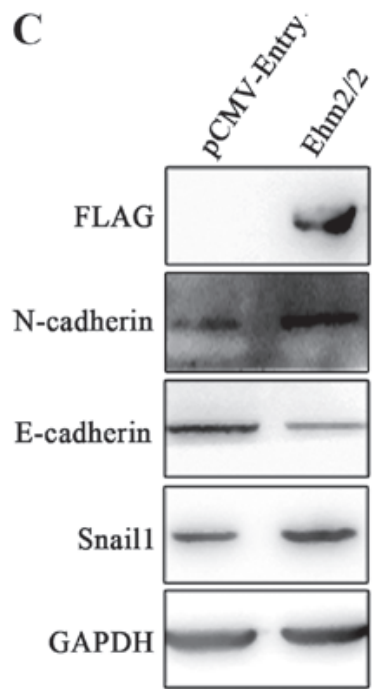

Figure 5. Protein expression levels of the epithelial-mesenchymal transition-related markers, E-cadherin, N-cadherin, and Snaill were assessed by western blot analysis. (A) Ehm2/1-overexpressing cells, (B) cells in which Ehm2/1 was knocked down, (C) Ehm2/2-overexpressing cells.

whereas the expression of E-cadherin was elevated (Fig. 5A). In line with this result, the knockdown of Ehm2/1 resulted in the downregulation of E-cadherin expression and in the upregulated expression of both $\mathrm{N}$-cadherin and Snail1 (Fig. 5B). However, the overexpression of Ehm2/2 resulted in the increased expression of $\mathrm{N}$-cadherin and Snail1, and in the decreased expression of E-cadherin (Fig. 5C). These results indicate that Ehm2/1 inhibits and Ehm2/2 promotes EMT in lung cancer cells.

Ehm2/1 transcripts are positively associated with and Ehm2/2 transcripts are negatively associated with the longer survival of patients with lung cancer. In order to further elucidate the clinical implication of Ehm2 variants in lung cancer, we analyzed the association of Ehm2/1 (Affy ID 220161_s_at) and Ehm2/2 (Affy ID 233098_s_at) with patient survival using Kaplan-Meier survival analysis (http://kmplot.com/analysis/). Lower expression levels of Ehm2/1 were associated with a shorter OS ( $\mathrm{P}=6.3 \mathrm{e}-06$; Fig. 6A) and PFS ( $\mathrm{P}=0.027$; Fig. 6B). A lower expression of Ehm2/1 was also associated with a poorer PPS ( $\mathrm{P}=0.0065$; Fig. $6 \mathrm{C}$ ). In contrast to Ehm2/1, a higher Ehm $2 / 2$ expression was observed in patients who had a poorer OS ( $\mathrm{P}=0.023$; Fig. $6 \mathrm{D})$ and $\mathrm{PFS}(\mathrm{P}=0.58$; Fig. $6 \mathrm{E})$. The elevated Ehm2/1 expression was also associated with a poor PPS ( $\mathrm{P}=0.032$; Fig. 6F). Taken together, these results indicate that Ehm2/1 inhibits and Ehm2/2 promotes disease progression and the relapse of lung cancer.

\section{Discussion}

In the current study, we demonstrated that two transcript variants of Ehm2 had distinct expression patterns in lung adenocarcinoma and had a different association with patient survival in lung cancer. We further demonstrated that the two transcript variants of Ehm2 had opposite effects on the functions of A549 cells and EMT in A549 cells. Our data 

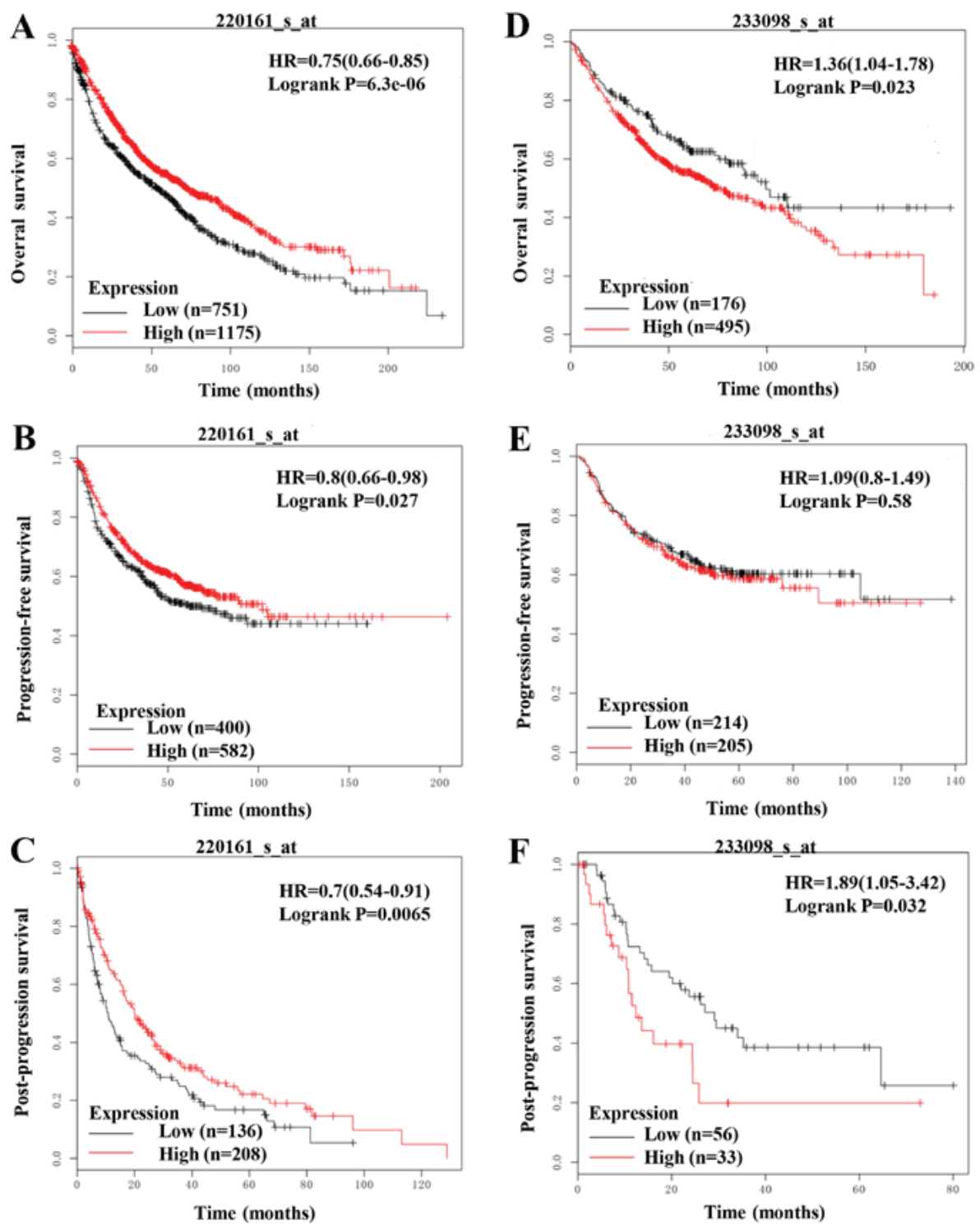

Figure 6. Association between Ehm2 transcript variants expression and survival of patients with lung cancer was analyzed by Kaplan-Meier analysis. Ehm2/1 expression and (A) overall survival, (B) progression-freesurvival, (C) and post-progression survival. (D-F) Association between Ehm2/2 expression and clinical outcome, as analyzed for (D) overall survival, (E) progression-free survival and (F) post-progression survival.

suggest that Ehm2/1 is a putative tumor suppressor during the disease progression and metastasis of lung cancer, while Ehm $2 / 2$ may have an opposite function.

The roles played by Ehm2 in cancer appear to be controversial. As a metastasis-associated protein, Ehm2 has been reported to be highly expressed in prostate and breast cancers, which is associated with disease progression and metastasis $(13,14)$. On the other hand, Ehm 2 has been shown to exert an inhibitory effect on the migration of HeLa cells by the regulation of cell morphology and cohesion as a downstream molecule of CRB3A (25). In fact, Ehm2 in these studies should be considered as total Ehm2, as the anti-Ehm2 antibody used in these studies recognizes an internal region of Ehm2, which is a sequence conserved in both Ehm 2/1 and Ehm2/2. However, which transcript variants dominate in these processes has yet to be elucidated. In this study, we used different antibodies specific to Ehm2/1 and Ehm2/2 to evaluate the expression and functions of these transcript variants in lung adenocarcinoma. In the present study, a decreased expression of Ehm2/1 was found in lung cancers, while an upregulation of Ehm $2 / 2$ was observed in the tumors. Significant associations were found between the reduced transcript levels of Ehm $2 / 1$ and poorer OS, PFS and PPS, while higher transcript levels of Ehm2/2 were associated with a poorer OS and PPS. Our results indicate that the two transcript variants have differential expression patterns and opposing functions in lung cancer development.

In line with their clinical implication, our in vitro experiments revealed that Ehm2/1 and Ehm2/2 have opposite effects on the functions of human lung adenocarcinoma A549 cells. A significant reduction in cell growth, migration and invasion in vitro was observed in Ehm2/1-overexpressing A549 cells. By contrast, Ehm2/1 knockdown led to a significant increase in the growth, migration and invasion of A549 cells. The overexpression of Ehm $2 / 2$ markedly promoted the migration and invasion of A549 cells. Our data suggest that Ehm2/1 is a putative suppressor of disease progression in lung cancer, while Ehm2/2 may play an opposing role. In fact, it is well known that different transcript variants of a gene may 
have different or opposing biological functions. For example, human osteopontin (OPN), has the 3 transcript variants of OPN-a (full-length form), OPN-b (lacking exon 5) and OPN-c (lacking exon 4). Chae et al demonstrated that OPN-a and OPN-b were predominantly expressed in hepatocellular carcinoma tissues, while OPN-c was expressed in normal liver tissues. The overexpression of OPN-a and OPN-b promoted the migration of Hep3B cells, while OPN-c exerted minimal effects. However, OPN-c inhibited the migrate of SK-Hep1 cells, while OPN-a overexpression had no effect (26).

Ehm2 contains a conserved FERM domain that is involved in the interaction between cytoplasmic proteins and transmembrane proteins via its FERM domain (27). Laprise et al revealed that Yurt, the Drosophila orthologue of Ehm2, can bind to Crb through its FERM domain and be recruited to the apical membrane (28). EPB41L5, a protein sharing a high homology with Ehm2, has been shown to bind with intracellular domain of Crumbs through its FERM domain, which is involved in the regulation of cell polarity (29). A predominant expression of endogenous Ehm2/1 at the cell membrane has been observed in a breast cancer cell line (MCF-7) in our previous study (22). In accordance with these results, the present study also revealed a predominant distribution of Ehm $2 / 1$ at the cell membrane in theA549 cells. By contrast, exogenous Ehm2/2 was widely distributed in the cells, including the cell membrane, cytoplasm and even the nucleus. As mentioned above, Ehm $2 / 2$ is longer than Ehm2/1 and has a longer C-terminal. Although structural analysis identified no specific domains in the C-terminus of Ehm2/2, we hypothesized that the C-terminus might be related to the different subcellular localization of the molecule, which is yet to be investigated. The present study clearly demonstrated that the two Ehm2 transcript variants have different expression patterns, different subcellular distributions and opposing biological functions. It is thus highly probable that different cellular distributions of Ehm2 variants may be one cause leading to varying biological functions. In our previous study, we reported that Ehm2/1 functions as a putative tumor suppressor in the disease progression of breast cancer (14). In our ongoing studies, we found that Ehm2/1 may stabilize E-cadherin by inhibiting its ubiquitination and degradation (unpublished data). It is therefore argued that different distributions of Ehm2 variants in different cellular compartments enable them to exert biological functions via different signaling pathway. This would be an exciting area to explore in the future. The current research findings also raised another question as to whether the two transcript variants interact with each other. Presently, there is no direct and solid evidence to suggest that the two variants do interact. However, in our humble opinion, and from these protein sequences, the two transcript variants of Ehm 2 are unlikely to interact with each other. Indeed, to the best of our knowledge, there has been no report to date showing FERM proteins interacting with each other, a notion worthwhile to explore in the future.

EMT is a highly regulated process, which is associated with the enhanced migration and invasion of cancerous cells, thus contributing to the local invasion and metastasis of cancer cells. EMT is typically defined by the loss of the epithelial phenotype and the acquiring of mesenchymal characteristics, leading to reduced cell-cell adhesion, increased motility and invasiveness and strengthened survival (30-32). Our previous study demonstrated that Ehm2/1 overexpression upregulated E-cadherin expression and decreased the migration of MCF-7 cells (14). The results of the present study also demonstrated that Ehm 2/1 and Ehm2/2 regulated the migration and invasion of A549 cells, key hallmarks of EMT. These findings led us to hypothesize that Ehm2/1 and Ehm2/2 may also regulate EMT in A549 cells. In line with this hypothesis, we found that Ehm2/1 inhibited EMT, while Ehm2/2 promoted EMT. This finding is indeed noteworthy and is in accordance to an ongoing finding in breast cancer, where we demonstrated that Ehm2/1 may stabilize E-cadherin by inhibiting its ubiquitination and degradation. These two studies were on different types of cancer and used different cell types, and suggest that Ehm2 is more widely involved in EMT in different cancer types and that different variants may have contrasting effects on EMT; these findings warrant further investigation in the future.

MMPs are a family of enzymes involved in the degradation of the extracellular matrix (ECM) and play important roles in tumor invasion and metastasis (33). High levels of MMP2 expression are often associated with metastasis (34). Studies have demonstrated that MMP2 leads to EMT via the proteolytic degradation of epithelial cell junctional proteins in carcinomas (35). MMP2 and MMP9, also known as gelatinases, play an important role for cleaving type I, IV collagen and contribute to the process of metastasis (36). In this study, in accordance with the data obtained from cell invasion assay, both the knockdown of Ehm2/1 and the overexpression of Ehm2/2 increased the activity of MMPs, including MMP2 and MMP9, suggesting that the regulation of MMPs in a different manner by different Ehm2 variants is another mechanism underlying their role in cancer metastasis. From this point of view, it is very important to compare the two Ehm 2 variants in primary and matched metastatic cancers. Unfortunately, our collection was small and only had one metastatic cancer out of the 15 lung cancer tissues making it difficult to draw a clear conclusion. However, we are currently collecting a larger tissue cohort, and hopefully with a longer follow-up of the patients, we would be in a position to conduct the comparison in near future.

In conclusion, the present study demonstrated that the Ehm2 variant, Ehm2/1, was downregulated in lung adenocarcinoma tissues and that these lower levels of Ehm2/1 were associated with a poorer survival. In vitro cell function assays indicated Ehm2/1 acted as an anti-oncogene in lung adenocarcinoma cells. This study also demonstrated that the other variant, Ehm2/2, had a contrasting pattern of expression from that of Ehm2/1, namely that it was upregulated in lung adenocarcinoma cells and higher levels of Ehm2/2 were associated with a poorer clinical outcome of patients with lung cancer. Cell function assays indicated that Ehm $2 / 2$ acted as an oncogene in lung adenocarcinoma cells. Moreover, Ehm2/1 inhibited the invasion and migration of lung cancer cells via the regulation of EMT, while Ehm2/2 increased the invasiveness and motility of lung cancer cells by promoting EMT. Our research provides new evidence that different transcript variants of a gene can play different, even opposing, roles. It will be necessary to validate these findings in in vivo models and accordingly we are currently 
preparing recombinant lentivirus for overexpressing Ehm $2 / 2$ and knocking down Ehm2/1 to order implement an orthotopic xenografts tumor model in BALB/c nude mice. Hopefully, these future investigation using in vivo models and indeed a larger clinical cohort would assist in answering some of questions relating to distant metastasis. Collectively, the current results from clinical samples and cellular experiments are sufficient to arrive at the conclusions that the two Ehm2 variants have contrasting expression pattern in human lung adenocarcinoma and exert contrasting biological functions in lung cancer cells. The variants have important bearing to the disease progression of lung adenocarcinoma.

\section{Acknowledgements}

The authors would also like to thank Professor Gong Liping of Capital Medical University for analyzing the results of immunohistochemistry of the tissue microarrays.

\section{Funding}

This study was supported by the National Natural Science Foundation of the People's Republic of China (grant no. 81672726).

\section{Availability of data and materials}

The corresponding experimental data of the present study are available from the corresponding author on reasonable request.

\section{Authors' contributions}

SL performed most of the experiments with assistance from JM, YS, SC and BL, and wrote the manuscript. XZ and MH provided the lung cancer tissue samples from the Department of Thoracic Surgery, Beijing Xuanwu Hospital. HY and WGJ conceived and designed the study, and also provided supervision and critically revised the manuscript. SL and HY performed the analysis of the data and also wrote the manuscript. All authors have reviewed and approved the final manuscript.

\section{Ethics approval and consent to participate}

The procedures of the human tissues collection were reviewed and approved by the Ethics Committee of Xuanwu Hospital, Capital Medical University [approval no. (2014) 022].

\section{Patient consent for publication}

Not applicable.

\section{Competing interests}

The authors declare that they have no competing interests.

\section{References}

1. Tomasini P, Greillier L and Barlesi F: Advanced non-squamous non-small-cell lung cancer: Who and when should be biologically screened today? Tomorrow? Curr Respir Care Rep 2: 17-21, 2013.
2. Miller KD, Siegel RL, Lin CC, Mariotto AB, Kramer JL, Rowland JH, Stein KD, Alteri R and Jemal A: Cancer treatment and survivorship statistics, 2016. CA Cancer J Clin 66: 271-289, 2016.

3. Castells M, Thibault B, Delord JP and Couderc B: Implication of tumor microenvironment in chemoresistance: Tumor-associated stromal cells protect tumor cells from cell death. Int J Mol Sci 13: 9545-9571, 2012.

4. Keedy VL, Temin S, Somerfield MR, Beasley MB, Johnson DH, McShane LM, Milton DT, Strawn JR, Wakelee HA and Giaccone G: American Society of Clinical Oncology provisional clinical opinion: Epidermal growth factor receptor (EGFR) Mutation testing for patients with advanced non-small-cell lung cancer considering first-line EGFR tyrosine kinase inhibitor therapy. J Clin Oncol 29: 2121-2127, 2011.

5. Neel DS and Bivona TG: Resistance is futile: Overcoming resistance to targeted therapies in lung adenocarcinoma. NPJ Precis Oncol 1: 1-6, 2017.

6. Shimizu K, Nagamachi Y, Tani M, Kimura K, Shiroishi T, Wakana $S$ and Yokota J: Molecular cloning of a novel NF2/ERM/4.1 superfamily gene, ehm2, that is expressed in high-metastatic K1735 murine melanoma cells. Genomics 65 : 113-120, 2000.

7. Badouel C, Gardano L, Amin N, Garg A, Rosenfeld R, Le Bihan T and McNeill H: The FERM-domain protein Expanded regulates Hippo pathway activity via direct interactions with the transcriptional activator Yorkie. Dev Cell 16: 411-420, 2009.

8. Pearson MA, Reczek D, Bretscher A and Karplus PA: Structure of the ERM protein moesin reveals the FERM domain fold masked by an extended actin binding tail domain. Cell 101: 259-270, 2000

9. Marešová L, Vydarený T and Sychrová H: Comparison of the influence of small GTPases Arl1 and Ypt6 on yeast cells' tolerance to various stress factors. FEMS Yeast Res 12: 332-340, 2012.

10. Nakajima $\mathrm{H}$ and Tanoue T: Lulu2 regulates the circumferential actomyosin tensile system in epithelial cells through p114RhoGEF. J Cell Biol 195: 245-261, 2011.

11. Chauhan S, Pandey R, Way JF, Sroka TC, Demetriou MC, Kunz S, Cress AE, Mount DW and Miesfeld RL: Androgen regulation of the human FERM domain encoding gene EHM2 in a cell model of steroid-induced differentiation. Biochem Biophys Res Commun 310: 421-432, 2003.

12. Hashimoto Y, Shindo-Okada N, Tani M, Takeuchi K, Toma H and Yokota J: Identification of genes differentially expressed in association with metastatic potential of K-1735 murine melanoma by messenger RNA differential display. Cancer Res 56: 5266-5271, 1996.

13. Wang J, Cai Y, Penland R, Chauhan S, Miesfeld RL and Ittmann M: Increased expression of the metastasis-associated gene Ehm2 in prostate cancer. Prostate 66: 1641-1652, 2006.

14. Yu H, Ye L, Mansel RE, Zhang Y and Jiang WG: Clinical implications of the influence of Ehm2 on the aggressiveness of breast cancer cells through regulation of matrix metalloproteinase-9 expression. Mol Cancer Res 8: 1501-1512, 2010.

15. Stegle O, Drewe P, Bohnert R, Borgwardt K and Rätsch G: Statistical Tests for Detecting Differential RNA-Transcript Expression from Read Counts. Nat Preced 10: 4437-4448, 2010.

16. Lixia M, Zhijian C, Chao S, Chaojiang G and Congyi Z: Alternative splicing of breast cancer associated gene BRCA1 from breast cancer cell line. J Biochem Mol Biol 40: 15-21, 2007.

17. Black DL: Mechanisms of alternative pre-messenger RNA splicing. Annu Rev Biochem 72: 291-336, 2003.

18. Ghigna C, Giordano S, Shen H, Benvenuto F, Castiglioni F, Comoglio PM, Green MR, Riva S and Biamonti G: Cell motility is controlled by SF2/ASF through alternative splicing of the Ron protooncogene. Mol Cell 20: 881-890, 2005.

19. Moolenaar CE, Pieneman C, Walsh FS, Mooi WJ and Michalides RJ: Alternative splicing of neural-cell-adhesion molecule mRNA in human small-cell lung-cancer cell line H69. Int J Cancer 51: 238-243, 1992.

20. Sato H, Hiyama K, Ishioka S, Maeda H and Yamakido M: Alternative splicing, but not allelic loss, of the FHIT gene increases with development of lung cancer. Int J Oncol 15: 81-88, 1999.

21. Venables JP: Unbalanced alternative splicing and its significance in cancer. BioEssays 28: 378-386, 2006.

22. Yu H, Ge Z, Si Y, Chen G, Zhang Y and Jiang WG: The splice variant Ehm 2/1 in breast cancer MCF-7 cells interacted with $\beta$-catenin and increased its localization to plasma membrane. RSC Advances 6: 78436-78444, 2016. 
23. Yuan JS, Reed A, Feng C and Stewart CN: Statistical analysis of real-time PCR data. BMC Bioinformatics 7: 85, 2006.

24. Gary R and Bretscher A: Ezrin self-association involves binding of an $\mathrm{N}$-terminal domain to a normally masked C-terminal domain that includes the F-actin binding site. Mol Biol Cell 6: 1061-1075, 1995

25. Loie E, Charrier LE, Sollier K, Masson JY and Laprise P: CRB3A Controls the Morphology and Cohesion of Cancer Cells through Ehm2/p114RhoGEF-Dependent Signaling. Mol Cell Biol 35: 3423-3435, 2015.

26. Chae S, Jun HO, Lee EG, Yang SJ, Lee DC, Jung JK, Park KC, Yeom YI and Kim KW: Osteopontin splice variants differentially modulate the migratory activity of hepatocellular carcinoma cell lines. Int J Oncol 35: 1409-1416, 2009.

27. Mori T, Kitano K, Terawaki S, Maesaki R and Hakoshima T: Crystallographic characterization of the radixin FERM domain bound to the cytoplasmic tail of adhesion molecule CD44. Acta Crystallogr Sect F Struct Biol Cryst Commun 63: 844-847, 2007.

28. Laprise P, Beronja S, Silva-Gagliardi NF, Pellikka M, Jensen AM, McGlade CJ and Tepass U: The FERM protein Yurt is a negative regulatory component of the Crumbs complex that controls epithelial polarity and apical membrane size. Dev Cell 11: 363-374, 2006.

29. Gosens I, Sessa A, den Hollander AI, Letteboer SJ, Belloni V, Arends ML, Le Bivic A, Cremers FP, Broccoli V and Roepman R: FERM protein EPB41L5 is a novel member of the mammalian CRB-MPP5 polarity complex. Exp Cell Res 313: 3959-3970, 2007.
30. Lamouille S, Xu J and Derynck R: Molecular mechanisms of epithelial-mesenchymal transition. Nat Rev Mol Cell Biol 15: 178-196, 2014.

31. Puisieux A, Brabletz T and Caramel J: Oncogenic roles of EMT-inducing transcription factors. Nat Cell Biol 16: 488-494, 2014.

32. Cervantes-Arias A, Pang LY and Argyle DJ: Epithelialmesenchymal transition as a fundamental mechanism underlying the cancer phenotype. Vet Comp Oncol 11: 169-184, 2013.

33. Jezierska A and Motyl T: Matrix metalloproteinase-2 involvement in breast cancer progression: A mini-review. Med Sci Monit 15: RA32-RA40, 2009.

34. Niemiec JA, Adamczyk A, Małecki K, Majchrzyk K and Ryś J: Relationships between immunophenotype, Ki-67 index, microvascular density, Ep-CAM/P-cadherin, and MMP-2 expression in early-stage invasive ductal breast cancer. Appl Immunohistochem Mol Morphol 20: 550-560, 2012.

35. Liu Y, Sun X, Feng J, Deng LL, Liu Y, Li B, Zhu M, Lu C and Zhou L: MT2-MMP induces proteolysis and leads to EMT in carcinomas. Oncotarget 7: 48193-48205, 2016.

36. John A and Tuszynski G: The role of matrix metalloproteinases in tumor angiogenesis and tumor metastasis. Pathol Oncol Res 7: 14-23, 2001. 